\title{
Dynamic metabolic modeling of a microaerobic yeast co-culture: predicting and optimizing ethanol production from glucose/xylose mixtures
}

Timothy J Hanly and Michael A Henson*

\begin{abstract}
Background: A key step in any process that converts lignocellulose to biofuels is the efficient fermentation of both hexose and pentose sugars. The co-culture of respiratory-deficient Saccharomyces cerevisiae and wild-type Scheffersomyces stipitis has been identified as a promising system for microaerobic ethanol production because S. cerevisiae only consumes glucose while $S$. stipitis efficiently converts xylose to ethanol.

Results: To better predict how these two yeasts behave in batch co-culture and to optimize system performance, a dynamic flux balance model describing co-culture metabolism was developed from genome-scale metabolic reconstructions of the individual organisms. First a dynamic model was developed for each organism by estimating substrate uptake kinetic parameters from batch pure culture data and evaluating model extensibility to different microaerobic growth conditions. The co-culture model was constructed by combining the two individual models assuming a cellular objective of total growth rate maximization. To obtain accurate predictions of batch co-culture data collected at different microaerobic conditions, the $S$. cerevisiae maximum glucose uptake rate was reduced from its pure culture value to account for more efficient S. stipitis glucose uptake in co-culture. The dynamic coculture model was used to predict the inoculum concentration and aeration level that maximized batch ethanol productivity. The model predictions were validated with batch co-culture experiments performed at the optimal conditions. Furthermore, the dynamic model was used to predict how engineered improvements to the S. stipitis xylose transport system could improve co-culture ethanol production.
\end{abstract}

Conclusions: These results demonstrate the utility of the dynamic co-culture metabolic model for guiding process and metabolic engineering efforts aimed at increasing microaerobic ethanol production from glucose/xylose mixtures.

Keywords: Co-culture, Cellulosic ethanol, Fermentation, Mathematical modeling, Saccharomyces cerevisiae, Scheffersomyces stipitis

\section{Background}

An essential component of the quest for energy independence is to develop renewable sources of energy via the conversion of plant biomass to liquid transportation fuels. Lignocellulosic biomass is a heterogeneous collection of polymers that compose plant cell walls, namely celluloses, hemicelluloses, pectins, lignins and proteoglycans [1]. The production of liquid fuels from biomass currently occurs in four major steps: pretreatment to make the feedstock

\footnotetext{
* Correspondence: henson@ecs.umass.edu

Department of Chemical Engineering, University of Massachusetts, Goessmann Lab 159, 686 N. Pleasant St, Amherst, MA 01003-3110, USA
}

more amenable to enzymatic degradation, hydrolysis of the cellulose and hemicellulose fractions to release sugar monomers and oligomers, fermentation of the released hexose and pentose sugars to produce fuels, and recovery of the fuels from the reactor bulk using separation technologies such as distillation $[2,3]$.

The fermentation of sugar mixtures that result from biomass hydrolysis is a significant bottleneck in the overall process. Few fermentative microbes that efficiently convert both hexose and pentose sugars to liquid fuels such as ethanol have been identified $[4,5]$. The majority of current research efforts are focused on engineering multiple metabolic functionalities, such as the introduction of exogenous

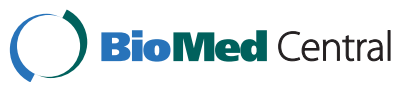


pentose consumption pathways, into a single organism [6]. However, this approach often results in conversion inefficiencies due to bottlenecks in metabolic pathways and may place a heavy metabolic burden on the organism $[7,8]$. In recent years, research has increasingly focused on the use of defined microbial consortia for biotechnology applications [9]. Microbial communities perform the task of biomass degradation in nature, albeit at a rate much slower than required for an industrial process [10]. Mixed cultures allow for the selection of microbes that are best suited for performing one task of the overall conversion process [11] and moves the engineering focus from introducing new functionalities to improving existing metabolic pathways. Other benefits of mixed culture systems include tunability and increased resistance to environmental stress [12,13].

Saccharomyces cerevisiae is a robust, budding yeast that has been widely used for fermentation of refined corn starch to fuel ethanol [14]. Because this yeast is Crabtreepositive, excess sugar that would otherwise overload its limited respiratory capacity overflows into the fermentative pathway. Thus, the yeast produces significant titers of ethanol when grown aerobically in batch culture [15]. However, S. cerevisiae is unable to utilize pentose sugars, such as xylose and arabinose, that result from the hydrolysis of hemicellulose. The engineering of pentose metabolism into the $S$. cerevisiae genome has been achieved, but problems with co-factor imbalances and gene expression have hindered the efficiency of these mutants [16].

Another species of yeast, Scheffersomyces stipitis (formerly known as Pichia stipitis), can natively ferment xylose to ethanol but it retains a preference for glucose as the carbon source. The growth and metabolite profile of this yeast is highly sensitive to the oxygenation level. Unlike $S$. cerevisiae, S. stipitis is a Crabtree-negative yeast that only produces ethanol under oxygen-limited conditions [17]. S. stipitis is among the most efficient native fermenters of xylose when grown under microaerobic culture conditions. In certain aeration regimes, however, this yeast can reassimilate ethanol while often simultaneously consuming other growth substrates [18]. Unlike most fermentative microorganisms, wild-type S. stipitis is unable to grow anaerobically. Insertion of the URA1 gene from $S$. cerevisiae has been shown to enable $S$. stipitis to grow anaerobically on glucose [19]. However, there are no known gene insertions that allow for S. stipitis anaerobic growth on xylose $[20,21]$. Thus, aeration level is a critical operating variable that must be tightly regulated to maximize xylose conversion to ethanol by wild-type S. stipitis.

S. cerevisiae and S. stipitis have been co-cultured for the production of ethanol from glucose and xylose mixtures [22-24]. In these studies, a respiratory-deficient strain of S. cerevisiae was used so the dissolved oxygen concentration could be more easily controlled at a level that was favorable for ethanol production by S. stipitis.
Because it lacks the respiratory capability of a wild-type strain, respiratory-deficient $S$. cerevisiae could not utilize non-fermentable carbon sources such as ethanol once glucose had been exhausted [25]. The inability of these strains to grow on the ethanol produced under certain microaerobic conditions resulted in high ethanol titers. In fact, co-culturing these two yeasts on a mixture of glucose and xylose has been shown to yield more ethanol than can be produced by either yeast alone [23].

Constraint-based analysis using genome-scale metabolic reconstructions is a widely used computational tool for predicting how fluxes through microbial metabolic pathways will respond to changes in the culture environment or gene deletions/insertions [26,27]. With the addition of substrate uptake kinetics and extracellular mass balances on growth-limiting substrates and metabolic byproducts, these steady-state models can be adapted to predict culture dynamics that are critical in batch and fed-batch fermentations [28-30]. Recently, constraints-based modeling has been applied to mixed-culture systems [31-33]. In addition to describing individual species metabolism, mixed-cultures models must account for possible interactions between the species as well as postulate a community objective that captures the combined metabolic behavior. While the assumption that each species attempts to maximize its own growth rate is most common, other community objectives that capture more complex behavior such as altruism can be employed [34].

In this study, we used dynamic flux balance analysis (DFBA) to drive the experimental optimization of ethanol production from a respiratory-deficient $S$. cerevisiae and wild-type S. stipitis co-culture growing microaerobically on a mixture of glucose and xylose. A dynamic model of S. stipitis metabolism was developed from a recently published genome-scale reconstruction [20] by estimating glucose and xylose uptake parameters from batch pure culture data. Because the respiratory-deficient $S$. cerevisiae mutant had non-specific genetic alterations, we considered several plausible modifications of the wild-type metabolic network [35] to develop a S. cerevisiae dynamic model consistent with batch pure culture data. Co-culture experiments demonstrated that $S$. cerevisiae competed less successfully for glucose than expected from combining the pure culture dynamic models under the community objective of total biomass maximization. A revised coculture model with reduced S. cerevisiae glucose uptake was shown to provide accurate predictions of batch coculture data over a range of microaerobic growth conditions. The experimentally validated model was used to optimize the batch ethanol productivity by adjusting the inoculum concentrations and the aeration level. Finally, the model was used to examine what modifications to the S. stipitis xylose transport system would yield the largest improvements in co-culture ethanol production. 


\section{Results and discussion}

\section{S. stipitis pure cultures}

Our initial dynamic model of $S$. stipitis metabolism did not include a balance on dissolved oxygen in the culture media. We were unable to satisfactorily fit this model to measured biomass and ethanol concentration profiles through adjustment of the sugar uptake rate parameters (see below). Because the dissolved oxygen (DO) concentration was above the assumed microaerobic value during the initial portion of the batch, we added the DO balance (8) to account for the apparent gas-liquid mass transfer limitations. The mass transfer coefficient $\left(k_{L} a\right)$ was determined from the gas sparge rate using a linear correlation (see Materials and Methods). Starting with literatures values when available [36], the uptake rate parameters for glucose (9), xylose (10) and oxygen (11) were determined by minimizing the leastsquares difference between the experimental and predicted concentration profiles. Parameter adjustments were made by trial-and-error using glucose, xylose, biomass, and ethanol concentration profile measurements collected from two microaerobic batch fermentations performed at air sparging rates of $25\left(k_{L} a=5.5 \mathrm{~h}^{-1}\right)$ and $50 \mathrm{cc} / \mathrm{min}\left(k_{L} a=10.1 \mathrm{~h}^{-1}\right)$. The resulting uptake parameters are compiled in Table 1.

The S. stipitis dynamic models with and without the DO balance (8) are compared in Figure 1 at an air sparging rate of $50 \mathrm{cc} / \mathrm{min}$ using initial conditions of $0.40 \mathrm{~g} / \mathrm{L}$ biomass, $15.7 \mathrm{~g} / \mathrm{L}$ glucose and $8.4 \mathrm{~g} / \mathrm{L}$ xylose. A $k_{L} a$ value of $10.1 \mathrm{~h}^{-1}$ was used in the DO balance, while the model without the balance used a constant DO value of 0.0072 $\mathrm{mM}$, the measured level at the end of the batch fermentation. The constant DO model could not reproduce the lag in ethanol production that resulted from the high level of dissolved oxygen present during the early stages of the fermentation. Additionally, the constant DO model produced lower biomass yields and higher ethanol yields than observed in experiment. Once the DO balance was added, the model could more accurately predict the time at which $S$. stipitis began microaerobic substrate consumption and ethanol production started to outpace biomass growth. The other $S$. stipitis pure culture

Table 1 Substrate uptake rate parameters for pure and co-culture dynamic flux balance models

\begin{tabular}{lll}
\hline Parameter & S. cerevisiae $\mathbf{3 1 1}$ & S. stipitis \\
\hline $\mathrm{V}_{\mathrm{g}, \max }(\mathrm{mmol} / \mathrm{gdw} / \mathrm{h})$ & 21.5 (pure culture) 18.5 (co-culture) & 6.5 \\
$\mathrm{~V}_{\mathrm{z}, \max }(\mathrm{mmol} / \mathrm{gdw} / \mathrm{h})$ & - & 5.5 \\
$\mathrm{~V}_{\mathrm{o}, \max }(\mathrm{mmol} / \mathrm{gdw} / \mathrm{h})$ & 2.5 & 11 \\
$\mathrm{~K}_{\mathrm{g}}(\mathrm{g} / \mathrm{L})$ & 0.5 & 1 \\
$\mathrm{~K}_{\mathrm{z}}(\mathrm{g} / \mathrm{L})$ & - & 0.25 \\
$\mathrm{~K}_{\mathrm{o}}(\mathrm{mM})$ & .005 & 0.0125 \\
$\mathrm{~K}_{\text {ieg }} / \mathrm{K}_{\text {iez }}(\mathrm{g} / \mathrm{L})$ & $10 /-$ & $10 / 4.5$ \\
$\mathrm{~K}_{\text {igz }}(\mathrm{g} / \mathrm{L})$ & - & 0.5 \\
\hline
\end{tabular}

experiment used for uptake parameter fitting of the variable DO model was performed with a $k_{L} a$ of $5.5 \mathrm{~h}^{-1}$ and initial conditions of $0.25 \mathrm{~g} / \mathrm{L}$ biomass, $16.8 \mathrm{~g} / \mathrm{L}$ glucose and $8.4 \mathrm{~g} / \mathrm{L}$ xylose (Figure 2A). As before, the model produced very accurate predictions of the glucose, xylose, biomass and ethanol concentration profiles with the uptake parameter values in Table 1 .

To examine extensibility of the $S$. stipitis dynamic model to different microaerobic conditions, batch experiments were performed at two additional $k_{L} a$ values and model predictions were generated with the same uptake parameter values listed in Table 1 . Figure $2 \mathrm{~B}$ shows a comparison of measured and predicted concentration profiles for a batch culture performed with a $k_{L} a$ of $7.6 \mathrm{~h}^{-1}$ and initial conditions of $0.25 \mathrm{~g} / \mathrm{L}$ biomass, $16.8 \mathrm{~g} / \mathrm{L}$ glucose and $8.0 \mathrm{~g} / \mathrm{L}$ xylose. The simulation results were generally satisfactory, although the model predicted slightly faster consumption of both substrates and small overprediction of biomass production throughout most of the batch. A second validation experiment was performed with a larger $k_{L} a$ of $12.6 \mathrm{~h}^{-1}$ and initial conditions of $0.2 \mathrm{~g} / \mathrm{L}$ biomass, $15.6 \mathrm{~g} / \mathrm{L}$ glucose and $8.6 \mathrm{~g} / \mathrm{L}$ xylose. As shown in Figure $2 \mathrm{C}$, the model adequately captured the fermentation dynamics throughout the first 15 hours but it was unable to predict the subsequent reassimilation of ethanol that is evident in the data.

Although the $S$. stipitis dynamic metabolic model generated satisfactory predictions over a range of microaerobic conditions, the model failed to capture the ethanol and biomass concentration profiles at higher aeration levels once glucose had been exhausted. Ethanol can be simultaneously produced and reassimilated at these higher $k_{L} a$ values [18], resulting in overprediction of ethanol secretion and underprediction of biomass formation by our model. This phenomenon is likely due to the relative ease at which ethanol diffuses across the plasma membrane [37], while the uptake of xylose has been shown to be facilitated by oxygen [38]. Since xylose was more energetically favorable, the LP solver returned a flux distribution with simultaneous xylose and ethanol uptake only if there was excess oxygen available beyond that required for xylose metabolism. Even in absence of xylose, the S. stipitis metabolic reconstruction predicted that $k_{L} a$ values greater than $34 \mathrm{~h}^{-1}$ would be required just to meet the ATP maintenance demand for growth on ethanol. By contrast, we observed ethanol assimilation in our experiments for $k_{L} a$ values as low as $12.6 \mathrm{~h}^{-1}$. Without altering the objective function or the ATP maintenance coefficient of the iBB814 model, the observed disparity between model and experiment cannot be resolved. Fortunately, the highest ethanol yields and titers observed experimentally were obtained at aeration levels under which ethanol assimilation did not occur. As a result, we do not consider $k_{L} a$ values above $\sim 10 \mathrm{~h}^{-1}$ in the remainder of the paper. 


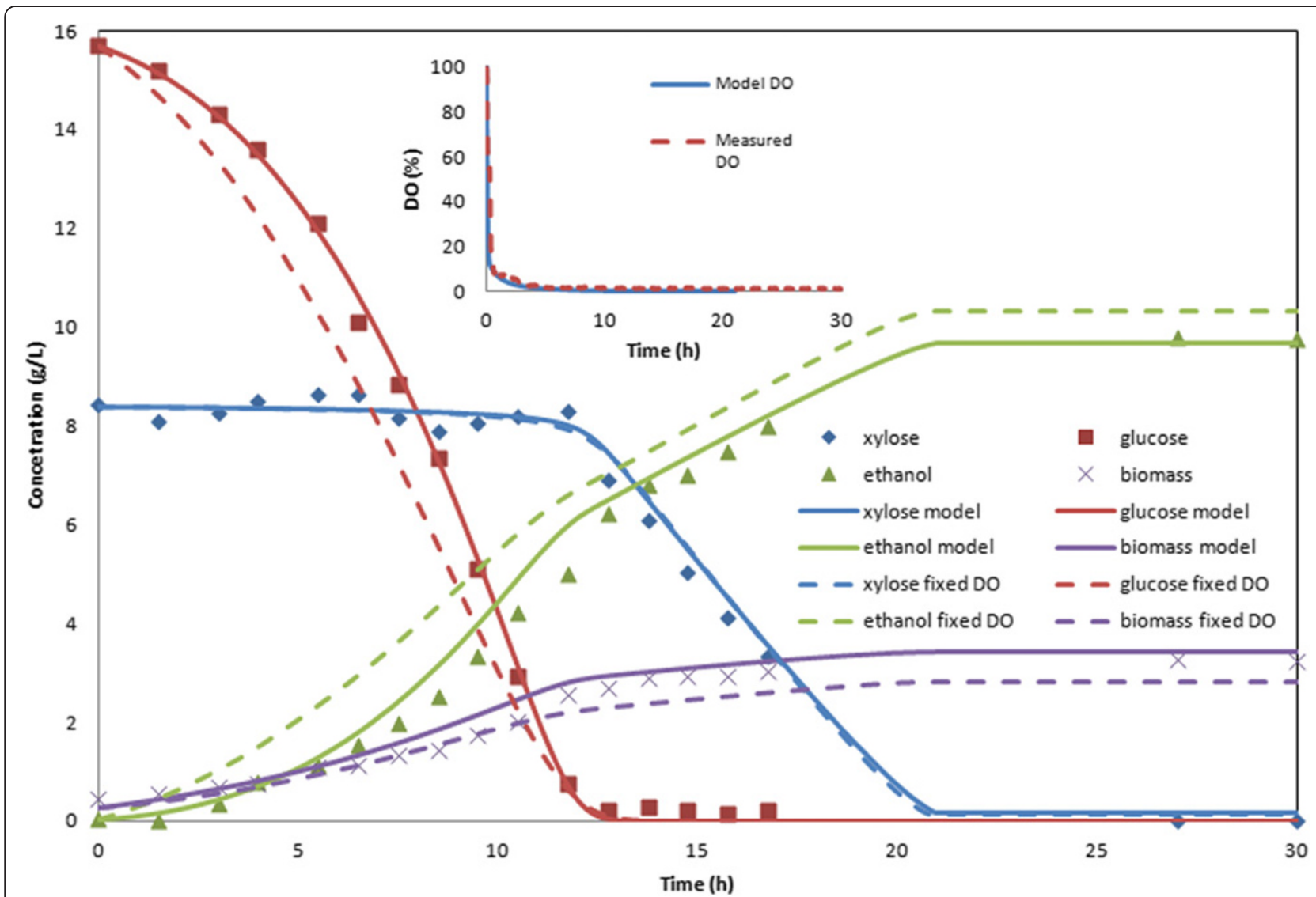

Figure 1 S. stipitis batch culture aerated at a $\boldsymbol{k}_{L} \boldsymbol{a}$ of $\mathbf{1 0 . 1} \mathrm{h}^{-1}$. Solid lines show dynamic model predictions when a balance on dissolved oxygen was included, while the dashed lines show dynamic model predictions at a fixed dissolved oxygen concentration of $0.00072 \mathrm{mM}$. Measured and predicted dissolved oxygen concentrations presented as percentage of the saturation value are shown in the inset.

\section{S. cerevisiae 311 pure cultures}

Because S. cerevisiae 311 was created by applying ethidium bromide to a wild-type strain, the genetic alterations that produced the respiratory-deficient mutant were non-specific. As a result, modifications to the wildtype iMM904 metabolic network necessary to describe $S$. cerevisiae 311 metabolism were unknown. We used the metabolic network model to identify putative gene knockouts consistent with the known alteration of mitochondrial DNA by ethidium bromide treatment [39] and to obtain predictions in agreement with our data. All the gene knockouts considered prevented growth on nonfermentable substrates and reduced oxygen demand. To determine the reactions to be deleted, model fluxes in the mitochondrial compartment were systematically set to zero until FBA simulations matched the $S$. cerevisiae 311 phenotype of lower biomass yields, higher ethanol yields and smaller oxygen demands than the wildtype strain. This process identified knockouts of genes encoding two enzymes: ubiquinol-6 cytochrome $\mathrm{c}$ reductase and mitochondrial cytochrome c oxidase. Modifications to these enzymes may well have occurred in
S. cerevisiae 311, as other respiratory-deficient S. cerevisiae mutants have been created through the direct removal of the encoding genes [40].

The S. cerevisiae 311 dynamic model was built on the modified iMM904 metabolic network with glucose uptake parameters estimated from anaerobic culture data and oxygen uptake parameters estimated from aerobic culture data with the glucose parameters fixed [41]. The resulting parameter values are listed in Table 1. Figure 3A shows a comparison of measured concentration profiles and model predictions for the anaerobic batch fermentation initiated with $0.28 \mathrm{~g} / \mathrm{L}$ biomass, $18.0 \mathrm{~g} / \mathrm{L}$ glucose and $1.8 \mathrm{~g} / \mathrm{L}$ residual ethanol from the preculture shake flask. Figure 3B shows results for the aerobic fermentation initiated with $0.20 \mathrm{~g} / \mathrm{L}$ biomass, $15.5 \mathrm{~g} / \mathrm{L}$ glucose and $1.8 \mathrm{~g} / \mathrm{L}$ ethanol. In each case, the dynamic model accurately predicted the entire ethanol profile and the glucose and biomass profiles in the second half of the batch. However, the model overpredicted glucose uptake and ethanol synthesis during the first half of the batch. While this discrepancy could have been attributable to the preculture cells used for inoculation being in 

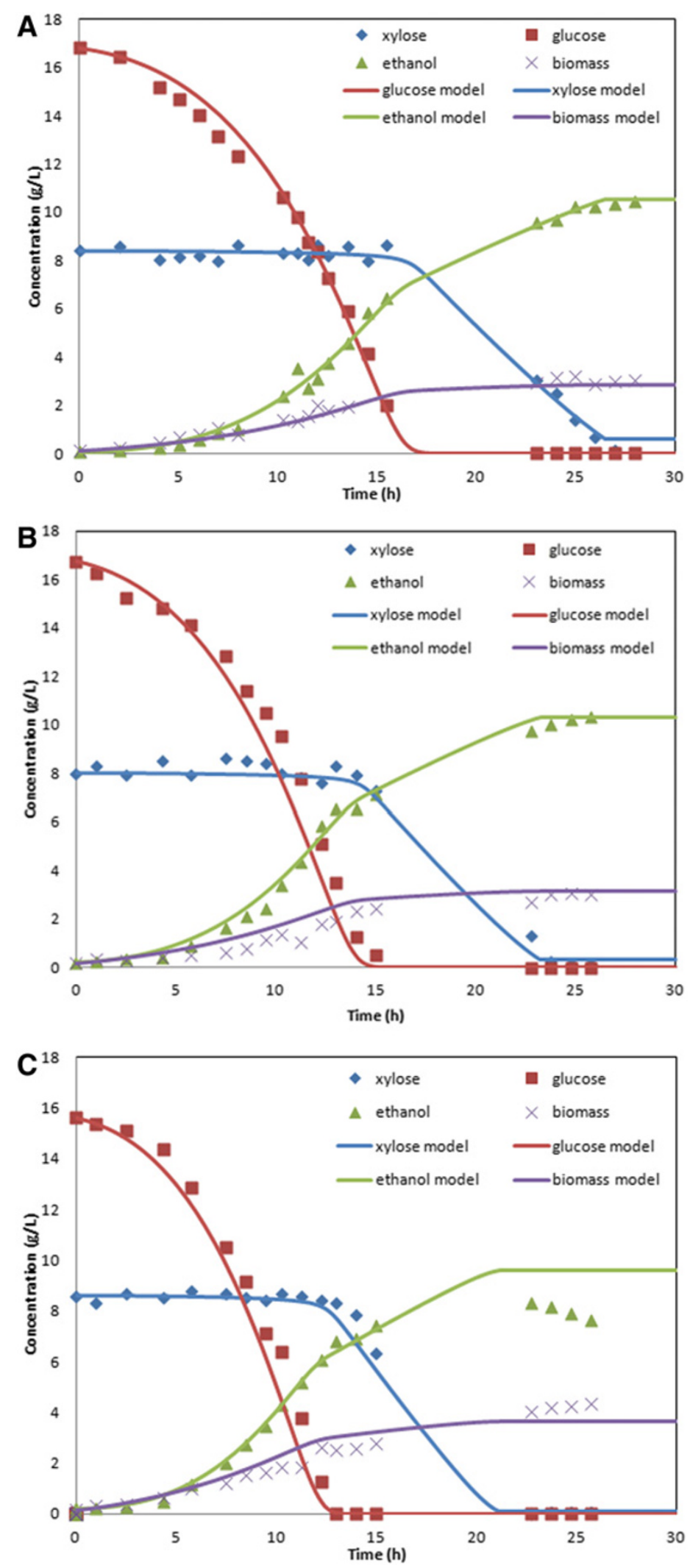

Figure 2 S. stipitis batch cultures aerated at a $k_{L} a$ of A) $5.5 \mathrm{~h}^{-1}$ B) $7.6 \mathrm{~h}^{-1}$ and C) $12.6 \mathrm{~h}^{-1}$. Data points are experimental measurements, while solid lines are dynamic model predictions 

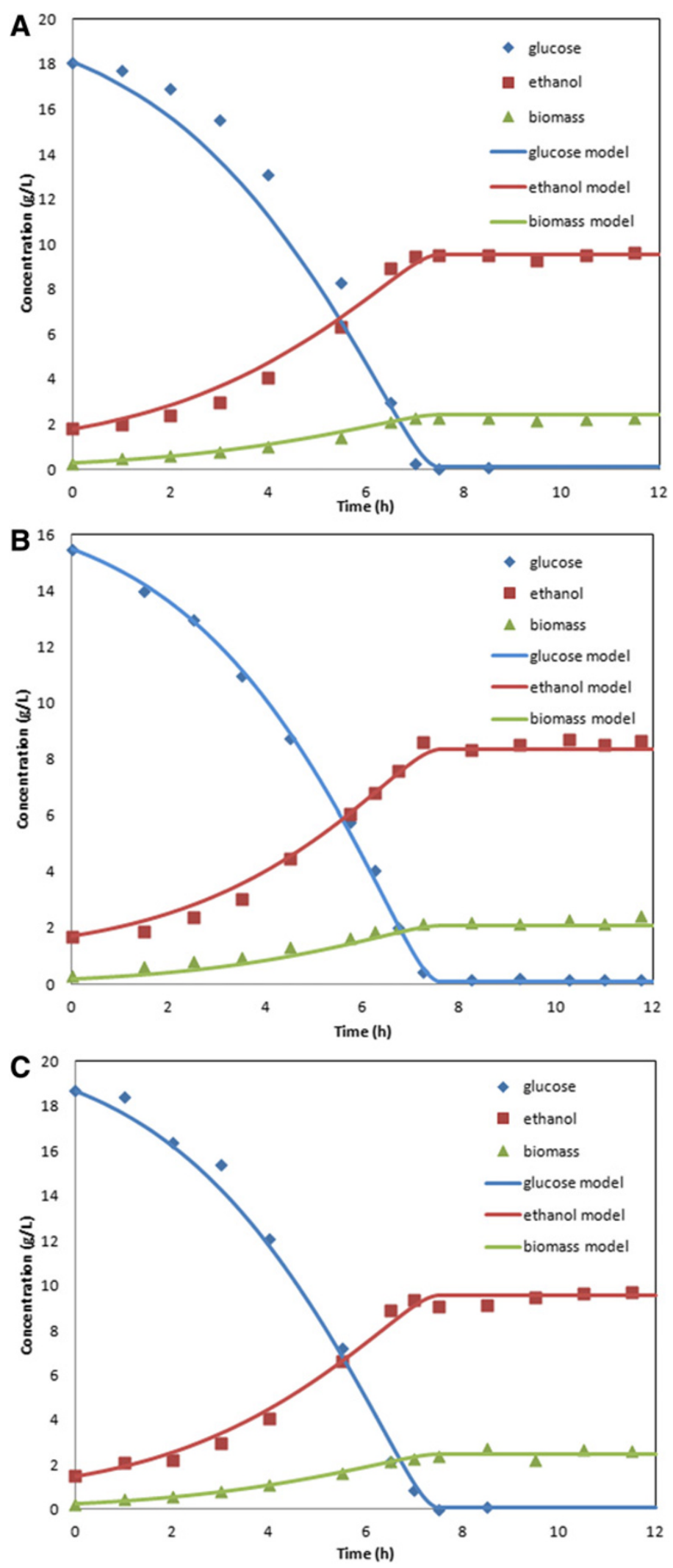

Figure 3 S. cerevisiae 311 batch cultures grown A) anaerobically B) aerobically and C) at a $k_{L} a$ of $5.5 \mathbf{h}^{-1}$. Data points are experimental measurements, while solid lines are dynamic model predictions. 
stationary rather than exponential phase [42], we were unable to verify this hypothesis through additional experiments in which the preculture cells were harvested earlier.

To examine extensibility of the $S$. cerevisiae dynamic model to different aeration levels, a microaerobic batch experiment was performed at a $k_{L} a$ of $5.5 \mathrm{~h}^{-1}$ and model predictions were generated with the same uptake parameter values listed in Table 1 . Figure $3 \mathrm{C}$ shows the results obtained for the fermentation initiated with $0.26 \mathrm{~g} / \mathrm{L}$ biomass, $18.7 \mathrm{~g} / \mathrm{L}$ glucose and $1.4 \mathrm{~g} / \mathrm{L}$ residual ethanol. The model produced excellent agreement with data, and only small deviations in the biomass concentration during the initial portion of the batch and in the ethanol concentration following glucose exhaustion were observed.

\section{Co-culture modeling}

A preliminary dynamic co-culture model was developed by direct combination of the two individual yeast models assuming no species interactions other than competition for glucose (see Materials and Methods). This approach resulted in faster glucose consumption and a higher final concentration of S. cerevisiae 311 than were observed in our microaerobic batch fermentations (not shown). We found that these discrepancies could be partially rectified by reducing the $S$. cerevisiae maximum glucose uptake rate from the pure culture value of $21.5 \mathrm{mmol} / \mathrm{gdw} / \mathrm{h}$ to $18.5 \mathrm{mmol} / \mathrm{gdw} / \mathrm{h}$. Otherwise, the substrate uptake parameters remained fixed at the pure culture values listed in Table 1.

Figure 4A shows a comparison of the resulting model predictions and experimental data collected for three batch fermentations at a $k_{L} a$ of $5.5 \mathrm{~h}^{-1}$ with an equal inoculum of $0.15 \mathrm{~g} / \mathrm{L}$ of each yeast species grown on 19.1 $\mathrm{g} / \mathrm{L}$ glucose and $9.7 \mathrm{~g} / \mathrm{L}$ xylose. Figure 4B shows corresponding results for a $k_{L} a$ of $10.1 \mathrm{~h}^{-1}, 0.10 \mathrm{~g} / \mathrm{L}$ of each yeast species, $16.5 \mathrm{~g} / \mathrm{L}$ glucose and $7.9 \mathrm{~g} / \mathrm{L}$ xylose. With the implemented change in the S. cerevisiae maximum glucose uptake rate, the dynamic co-culture model proved to be as accurate as the individual species models. The glucose consumption rate was slightly overpredicted during the initial portion of the batch at both aeration levels. Small discrepancies in the predicted biomass and ethanol concentrations were observed during the glucose consumption phase for $k_{L} a=5.5 \mathrm{~h}^{-1}$, while small but longer lasting deviations were apparent in the biomass and xylose concentrations at $k_{L} a=10.1 \mathrm{~h}^{-1}$.

To examine prediction accuracy of dynamic co-culture model at aeration levels not used for parameter adjustment, we performed three additional co-culture fermentations at a $k_{L} a$ of $7.6 \mathrm{~h}^{-1}$ with $0.145 \mathrm{~g} / \mathrm{L}$ of each yeast as the inoculum and initial sugar concentrations of $16.5 \mathrm{~g} / \mathrm{L}$ glucose and $8.5 \mathrm{~g} / \mathrm{L}$ xylose. The model and data comparisons shown in Figure $4 \mathrm{C}$ are qualitatively similar to those obtained at the other two aeration levels. The results in Figure 4 collectively demonstrate that the co-culture model provided satisfactory predictions over a range of microaerobic conditions corresponding to $k_{L} a<\sim 10 \mathrm{~h}^{-1}$.

\section{Inoculum optimization}

Following experimental validation, we utilized the dynamic co-culture model to computationally determine optimal bioreactor operating conditions for maximization of ethanol productivity. The productivity was defined as the final ethanol concentration divided by the batch time, which was chosen as the time at which the xylose concentration dropped below $0.5 \mathrm{~g} / \mathrm{L}$. Our simulations indicated that the aeration level had little effect on the ethanol titer but strongly affected the batch time due to the xylose consumption rate [38]. Our simulations also demonstrated that the relative amount of each yeast in the inoculum strongly affected the productivity through both the ethanol titer and the batch time. Therefore, ethanol productivity was optimized by adjusting the aeration level and inoculum concentrations. Rather than perform rigorous optimization [30], dynamic simulations were run with different combinations of the $k_{L} a$ value and the initial yeast concentrations, and the case that yielded the largest productivity was deemed the optimal solution. All simulations were performed with a mixture of $16.0 \mathrm{~g} / \mathrm{L}$ glucose and $8.0 \mathrm{~g} / \mathrm{L}$ xylose, while the total inoculum concentration was fixed at $1.0 \mathrm{~g} / \mathrm{L}$ to allow direct comparison of results for different inocula.

The $k_{L} a$ value was constrained to be below $10.1 \mathrm{~h}^{-1}$ since larger values had the potential to result in undesirable ethanol reassimilation by $S$. stipitis that was not captured by the co-culture model. Regardless of the inoculum, we found that increasing $k_{L} a$ improved productivity due to a decrease in batch time that resulted from enhanced xylose consumption. Consequently, the optimal solution was achieved at $k_{L} a=10.1 \mathrm{~h}^{-1}$, and the optimization problem was reduced to determining the inoculum concentrations. This result demonstrated the importance of developing improved metabolic reconstructions that more accurately predict the relationship between aeration level and the onset of ethanol reassimilation. Figure 5 shows the effect of the initial S. cerevisiae 311 concentration on the ethanol titer, batch time and ethanol productivity. The initial S. stipitis concentration is not shown since the total inoculum concentration was constrained to be $1.0 \mathrm{~g} / \mathrm{L}$. The ethanol titer increased with increasing S. cerevisiae concentration since this yeast converts glucose to ethanol at higher yields than $S$. stipitis. Conversely, the batch time decreased with increasing $S$. cerevisiae concentration because S. stipitis could better compete for glucose during the initial growth phase and the larger S. stipitis biomass concentration that resulted allowed for more rapid xylose consumption. These two competing effects produced a 

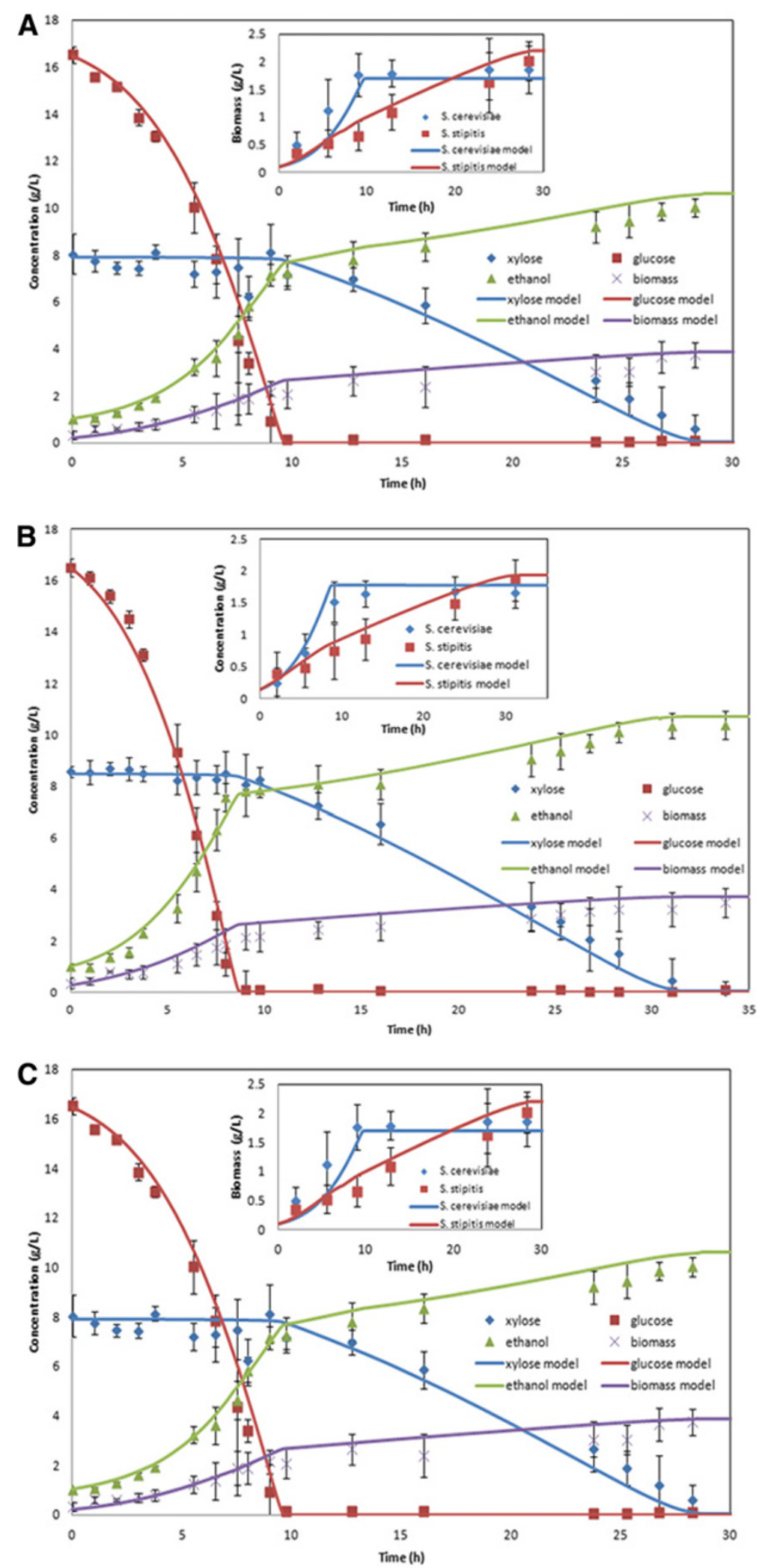

Figure 4 S. cerevisiae/S. stipitis batch co-cultures grown with an equal inoculum and aerated at A) $5.5 \mathrm{~h}^{-1}$ B) $9.6 \mathrm{~h}^{-1}$ and C) $7.6 \mathrm{~h}^{-1}$. Individual cell concentrations are shown in the inset. Dynamic model predictions are indicated by solid lines. Three separate fermentations were performed at each aeration level to compute average values indicated by the symbols and coefficients of variation indicated by the error bars. 


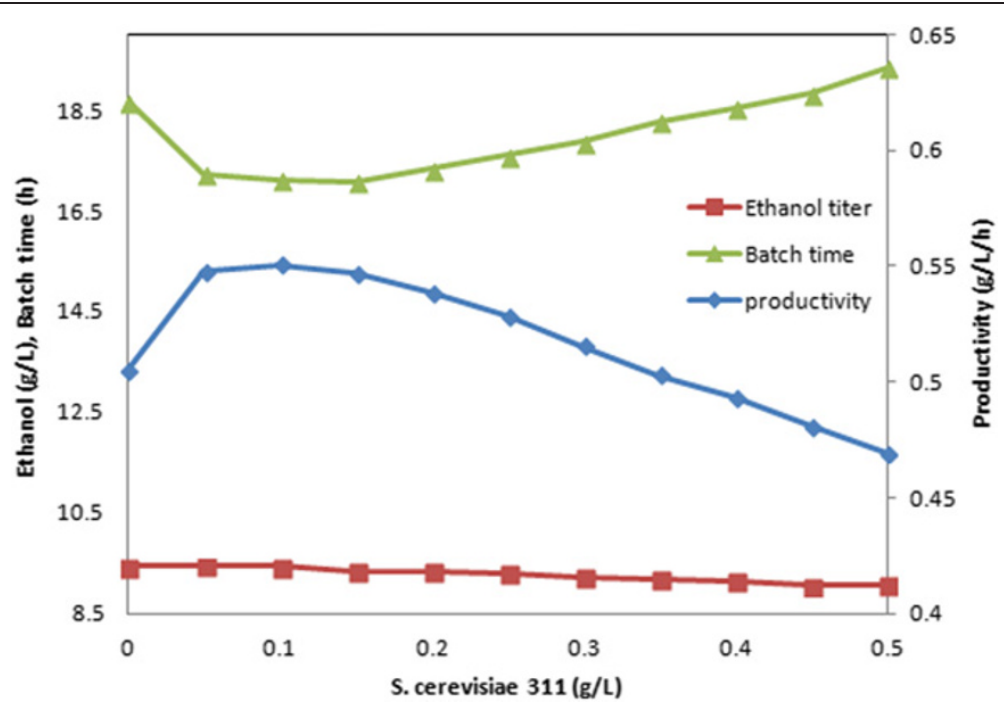

Figure 5 Predicted effect of the inoculum concentration on batch co-culture performance measures at a $k_{L} a$ of $10.1 \mathrm{~h}^{-1}$. The total inoculum concentration was fixed at $1 \mathrm{~g} / \mathrm{L}$.

clear optimum in the ethanol productivity at $0.1 \mathrm{~g} / \mathrm{L}$ S. cerevisiae and $0.9 \mathrm{~g} / \mathrm{L}$ S. stipitis.

To validate the model predictions, we performed three batch fermentations at the optimal conditions identified in silico. Figure 6 shows the averaged result of these experiments alongside the predicted growth and metabolite concentration curves. Although we were unable to obtain measurements during the first six hours, the optimized co-culture model produced excellent agreement with the measured concentration profiles after that time. The model predicted a final ethanol titer of $9.43 \mathrm{~g} / \mathrm{L}$ and a productivity of $0.55 \mathrm{~g} / \mathrm{L} / \mathrm{h}$, while the three fermentations were averaged to produce an ethanol titer of 9.07 $\mathrm{g} / \mathrm{L}$ and a productivity of $0.521 \mathrm{~g} / \mathrm{L} / \mathrm{h}$. According to our in silico analysis at $k_{L} a=10.1 \mathrm{~h}^{-1}$, the optimized coculture would outperform both a pure $S$. stipitis culture $(9.43 \mathrm{~g} / \mathrm{L}$ ethanol at a productivity of $0.505 \mathrm{~g} / \mathrm{L} / \mathrm{h})$ and a co-culture with equal inoculum $(9.07 \mathrm{~g} / \mathrm{L}$ ethanol at a productivity of $0.469 \mathrm{~g} / \mathrm{L} / \mathrm{h}$ ).

\section{In silico transporter engineering}

Our simulations demonstrated that S. stipitis xylose metabolism was the rate limiting process that limited coculture conversion efficiency. Moreover, xylose transport has been identified as the main bottleneck in pentose sugar metabolism with pure S. stipitis cultures [43]. Therefore, we used the dynamic co-culture model to predict the effects of modifying S. stipitis xylose transport parameters on ethanol productivity. To model engineering of the associated transport proteins, the nominal values listed in Table 1 for the maximum xylose uptake rate $\left(v_{z, \max }\right)$, the xylose uptake saturation constant $\left(K_{z}\right)$, and the glucose inhibition constant for xylose uptake $\left(K_{i g z}\right)$ were perturbed both upward and downward to determine their impact. The ethanol inhibition constant for xylose uptake $\left(K_{\text {iez }}\right)$ was excluded from detailed analysis because the ethanol concentrations achieved in silico were too small to cause significant xylose uptake inhibition. For the remaining three parameters, scaled sensitivity coefficients were calculated as:

$$
S=\frac{\bar{p}}{\bar{y}} \cdot \frac{\Delta y}{\Delta p}
$$

where $\bar{p}$ is the nominal parameter value, $\Delta \mathrm{p}$ is the parameter change, $\bar{y}$ is the optimal ethanol productivity obtained with the nominal parameter value, and $\Delta \mathrm{p}$ is the predicted change in the ethanol productivity that results from the parameter change. All simulations were initialized with $16 \mathrm{~g} / \mathrm{L}$ glucose and $8 \mathrm{~g} / \mathrm{L}$ xylose at the optimum $k_{L} a=10 \mathrm{~h}^{-1}$ and inoculum of $0.1 \mathrm{~g} / \mathrm{L}$ S. cerevisiae 311 and $0.9 \mathrm{~g} / \mathrm{L}$ S. stipitis.

Figure 7 shows the ethanol productivities and scaled sensitivity coefficients obtained when $v_{z \text {,max }}$ was changed upward and $K_{z}$ and $K_{i g z}$ were changed downward. Increasing the maximum xylose uptake rate offered the largest improvement in ethanol productivity as indicated by the relatively large sensitivity coefficients, with a productivity enhancement of $14 \%$ achieved with a $25 \%$ $v_{z, \text { max }}$ increase (Figure 7A). As indicated by the small sensitivity coefficients, more modest gains in ethanol productivity were predicted for decreases in the xylose uptake saturation constant (Figure 7B) and the glucose inhibition constant for xylose uptake (Figure 7C). However, complete elimination of S. stipitis diauxic growth by removal of the glucose inhibition term from the 


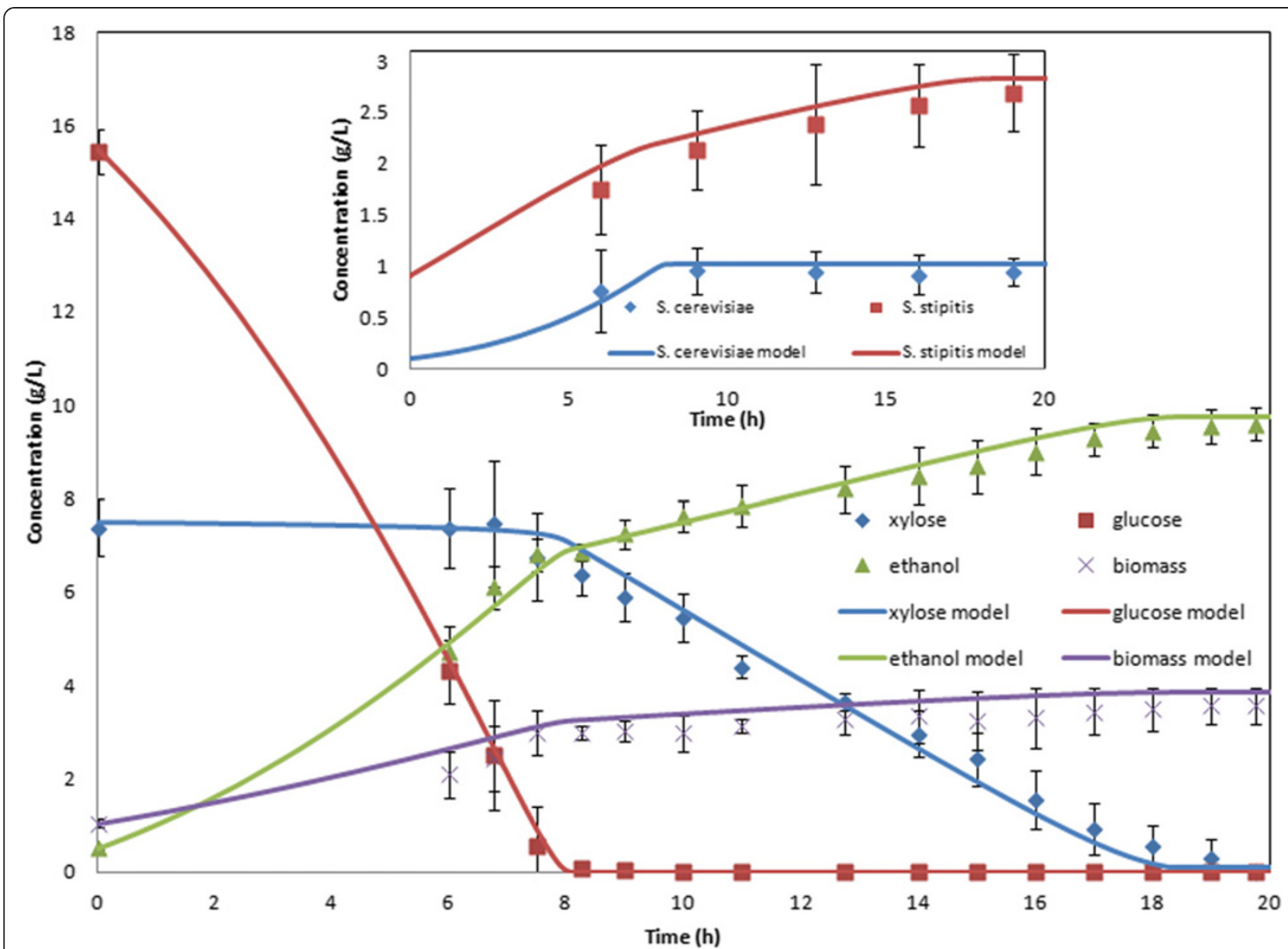

Figure 6 S. cerevisiae/S. stipitis batch co-cultures grown at the optimal conditions identified in silico. The co-culture was inoculated with $0.1 \mathrm{~g} / \mathrm{L}$ S. cerevisiae 311 and $0.9 \mathrm{~g} / \mathrm{L}$ S. stipitis and aerated at a $k_{L} a$ of $10.1 \mathrm{~h}^{-1}$. Individual cell concentrations are shown in the inset. Dynamic model predictions are indicated by solid lines. Three separate fermentations were performed to compute average values indicated by the symbols and coefficients of variation indicated by the error bars.

xylose uptake expression resulted in a large productivity enhancement of $85 \%$ (not shown). The simulated parameter changes could potentially be realized through protein engineering of the sugar transporters [44,45]. Because many S. stipitis transporters uptake both glucose and xylose [36], a complementary approach could be overexpression of specific transporters with more favorable xylose uptake characteristics and downregulation or deletion of less favorable transporters [46].

\section{Conclusions}

Due to the ability of Scheffersomyces stipitis to efficiently convert pentose sugars to ethanol, co-cultures of S. stipitis and respiratory-deficient Saccharomyces cerevisiae are promising for renewable ethanol production from glucose/xylose mixtures $[23,47]$. Starting from genome-scale metabolic reconstructions of the individual yeasts, we developed a dynamic metabolic model of $S$. cerevisiae/S. stipitis co-cultures that was capable of predicting microaerobic metabolism in batch culture. As a first step towards co-culture modeling, dynamic models were developed for the individual yeasts using data from pure culture experiments. The Crabtree-negative yeast $S$. stipitis was shown to be very sensitive to the aeration level such that the incorporation of a dissolved oxygen balance was necessary to capture non-microaerobic oxygen levels during the initial batch phase and the subsequent switch from respiratory and to fermentative growth. The S. stipitis dynamic model was able to accurately reproduce measured glucose, xylose, biomass and ethanol concentration profiles over a range of microaerobic growth conditions characterized by oxygen-liquid mass transfer coefficient $\left(k_{L} a\right)$ values below $\sim 10 \mathrm{~h}^{-1}$. However, the S. stipitis model was unable to capture ethanol reassimilation observed at higher oxygenation levels due to model energetics that required a $k_{L} a$ greater than $34 \mathrm{~h}^{-1}$ just to meet the ATP maintenance demand for growth on ethanol. Therefore, subsequent co-culture experiments and simulations were 

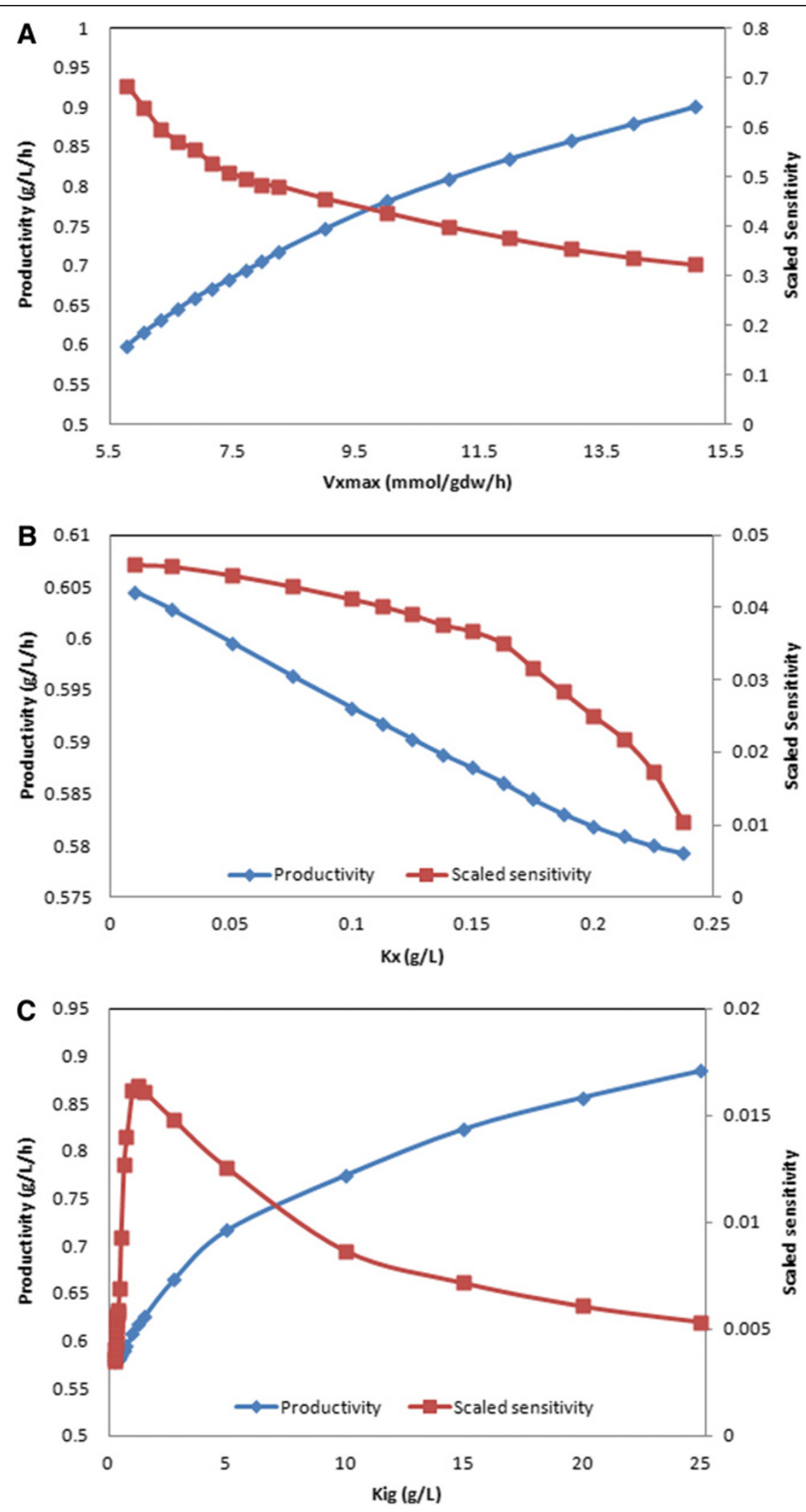

Figure 7 Ethanol productivities and scaled sensitivity coefficients predicted by varying S. stipitis model parameters for the A) xylose maximum uptake rate $\left(v_{z, \max }\right)$ B) xylose uptake saturation constant $\left(K_{z}\right)$ and C) glucose inhibition constant for xylose uptake. 
restricted to microaerobic conditions with $k_{L} a$ values less than $\sim 10 \mathrm{~h}^{-1}$.

The procedure used to create the respiratory-deficient strain S. cerevisiae 311 introduced unknown genetic alterations that could not be directly implemented in the wildtype metabolic network. We used flux balance analysis to screen putative gene knockouts in the mitochondrial compartment that would reproduce the S. cerevisiae 311 phenotype of lower biomass yields, higher ethanol yields and smaller oxygen demands than the wild-type strain. The best agreement with S. cerevisiae 311 pure culture data was obtained for deletions of two genes that encode the ubiquinol- 6 cytochrome c reductase and mitochondrial cytochrome c oxidase enzymes involved in the electron transport chain. With the fluxes through the two reactions catalyzed by these enzymes constrained to zero, the oxygen demand was greatly reduced because oxygen was not needed to serve as a final electron acceptor for ATP synthesis. These results were consistent with more directed genetic engineering efforts to create respiratorydeficient $S$. cerevisiae mutants through direct removal of these two genes [40].

A preliminary version of the dynamic co-culture model was developed by directly combining the dynamic models of the two yeast species under the assumption that each species attempted to maximize its individual growth rate and the only interspecies interaction was competition for glucose substrate. By comparison of model predictions to batch co-culture data collected at several microaerobic conditions, we found that the preliminary model overpredicted the glucose consumption and $S$. cerevisiae biomass formation rates. Much improved predictions were obtained by reducing the $S$. cerevisiae maximum glucose uptake rate from its pure culture value of $21.5 \mathrm{mmol} / \mathrm{gdw} / \mathrm{h}$ to $18.5 \mathrm{mmol} / \mathrm{gdw} / \mathrm{h}$. This parameter change suggested the presence of unmodeled species interactions that resulted in S. stipitis having an antagonistic effect on S. cerevisiae growth. Crabtree-negative yeasts like S. stipitis have an established advantage when competing against a Crabtree-positive yeast such as $S$. cerevisiae for the same growth-limiting substrate [48]. This effect is further magnified when the Crabtree-positive species is a respiratory deficient mutant [49]. One possible cause for this effect was the competition for nutrients other than glucose. Growth under nitrogen limited conditions has been shown to slow the uptake of glucose and other hexoses in wildtype $S$. cerevisiae [50]. Respiratory-deficient $S$. cerevisiae could be at a disadvantage in competing for nitrogen sources such as ammonium when grown with respiratory competent $S$. stipitis. Because sugar uptake parameters reflect the action of numerous transport systems, each having distinct mechanisms and affinities, the decrease in this parameter may indicate differences in transporter expression between pure and co-cultures of the two microbes.
Following experimental validation, the dynamic coculture model was used to compute the aeration level and initial cell concentrations that maximized batch ethanol productivity. Our in silico analysis suggested that the coculture should be operated at the largest possible $k_{L} a$ value before the onset of ethanol reassimilation by $S$. stipitis because this aeration level maximized the rate of xylose conversion to ethanol. Because the S. stipitis metabolic network model did not accurately predict this transition point, the optimization was constrained by experimental data such that the optimal solution was achieved at $k_{L} a=10.1 \mathrm{~h}^{-1}$. Optimization of the initial cell concentrations produced an inoculum with $90 \%$ S. stipitis and only $10 \%$ S. cerevisiae to form sufficient $S$. stipitis biomass following glucose exhaustion for efficient conversion of the remaining xylose. Experimental validation of the optimal solution showed that the co-culture model provided excellent agreement with measured concentration profiles. Compared to an unoptimized co-culture with equal initial cell concentrations, the optimized co-culture was shown experimentally to produce a $11 \%$ improvement in ethanol productivity. Additional in silico analysis indicated that the co-culture would yield higher ethanol productivities than a S. stipitis pure culture due to the higher glucose to ethanol conversion efficiency of $S$. cerevisiae.

The relatively slow conversion of xylose by S. stipitis is the main bottleneck that limits overall co-culture performance. The dynamic co-culture model was used to explore the potential impact of transporter engineering efforts aimed at enhancing xylose uptake by increasing the maximum xylose uptake rate, reducing xylose uptake saturation and reducing glucose inhibition of xylose uptake. Based on sensitivity analysis for moderate parameter changes, the maximum xylose uptake rate was predicted to yield the largest improvement in ethanol productivity. However, substantially larger improvements were predicted for complete elimination of glucose catabolite repression in S. stipitis because this modification eliminated the diauxic growth pattern and allowed xylose consumption to commence at the beginning of the batch. Taken together, our computational results suggest that an engineered S. stipitis strain which rapidly consumes xylose in the presence of glucose and does not reassimilate ethanol under microaerobic conditions would be an ideal candidate for enhancing coculture performance. Future work should focus on the creation of such $S$. stipitis strains as well as the model-based characterization of co-culture performance for growth on actual biomass hydrolysates with inhibitory compounds.

\section{Methods \\ Experimental}

The wild-type S. stipitis strain NRRL Y-7124 (ATCC 58376) was used in this study. S. cerevisiae 311 (ATCC 
42511), a mutant that was created by treating a wild-type strain with ethidium bromide [51], was chosen as the respiratory-deficient $S$. cerevisiae strain. Stocks of the two yeasts were stored at $4{ }^{\circ} \mathrm{C}$ on YM agar slants.

All pure and mixed cultures were performed in a synthetic yeast minimal medium [52]. The composition per liter of water was $1.00 \mathrm{~g} \mathrm{MgSO}_{4} \cdot 7 \mathrm{H}_{2} \mathrm{O}, 1.10 \mathrm{~g} / \mathrm{L} \mathrm{KCl}, 0.15 \mathrm{~g}$ $\mathrm{CaCl}_{2} \cdot 2 \mathrm{H}_{2} \mathrm{O}, 1.00 \mathrm{~g}\left(\mathrm{NH}_{4}\right)_{2} \mathrm{HPO}_{4}, 8.75 \mathrm{~g} / \mathrm{L}\left(\mathrm{NH}_{4}\right)_{2} \mathrm{SO} 4$, $60.3 \mathrm{mg}$ myo-inositol, $30.0 \mathrm{mg}$ Ca-panthothenate, $6.0 \mathrm{mg}$ thiamine- $\mathrm{HCl}, 1.5 \mathrm{mg}$ pyridoxine- $\mathrm{HCl}, 0.03 \mathrm{mg}$ biotin, $10.6 \mathrm{mg} \mathrm{MnSO}_{4} \cdot \mathrm{H}_{2} \mathrm{O}$, $9.0 \mathrm{mg} \mathrm{ZnSO}_{4} \cdot 7 \mathrm{H}_{2} \mathrm{O}$, $5.0 \mathrm{mg}$ $\mathrm{FeSO}_{4} \cdot 7 \mathrm{H}_{2} \mathrm{O}$, and $2.4 \mathrm{mg} \mathrm{CuSO}_{4} \cdot 2 \mathrm{H}_{2} \mathrm{O}$. Pre-cultures in media containing $20 \mathrm{~g} / \mathrm{L}$ glucose and $20 \mathrm{~g} / \mathrm{L}$ xylose for S. cerevisiae and S. stipitis, respectively, were grown at $30^{\circ} \mathrm{C}$ for 36 hours on a shake table set at 175 RPM. The inoculum concentration for each experiment was determined by calculating the volume of preculture required to obtain the target initial concentration of each cell type using the measured biomass concentration in the shake flask media.

All fermentations were performed in a HEL BioX array of 4. $250 \mathrm{~mL}$ vessels situated in a shared block that provided both electric heat and independent magnetic agitation (HEL Group Ltd., Barnet, UK). Electrochemical probes monitored the dissolved oxygen and $\mathrm{pH}$ in each vessel, while individual thermocouples recorded the media temperatures. Bioreactor cultivations were performed at a constant temperature of $30^{\circ} \mathrm{C}$ and $\mathrm{pH}$ of 5 , the optimal growth conditions for each yeast species [53]. The $\mathrm{pH}$ in each vessel was controlled by the automatic addition of $1 \mathrm{~N}$ sulfuric acid or $2 \mathrm{~N} \mathrm{NaOH}$. Glucose and xylose were autoclaved separately and added to the growth media in the amounts indicated for each experiment. Antifoam A was added to the reactors as necessary to prevent foaming.

Aeration of culture media was found to be a crucial operating variable. The agitation speed was held constant at 500 RPM for both pure and mixed culture fermentations. The gas flow rate into each reactor was altered according to the aeration level required for each experiment. A linear relationship between the gas sparge rate and the gasliquid oxygen mass transfer coefficient $\left(k_{L} a\right)$ was determined using the static gassing out method [54]. Purely aerobic cultures were aerated with pure oxygen, while microaerobic fermentations were aerated with house air passed through a HEPA-VENT filter (Whatman Ltd., Kent, UK).

Total cell weight was measured using a correlation between OD595 measured on a WPA UV1101 Biotech Photometer (Biochrom Ltd., Cambridge, UK) and dry cell weight. Cell counts of $S$. cerevisiae and S. stipitis in coculture were performed on a hemacytometer in triplicate and averaged. A typical cell count considered approximately 50 S. cerevisiae cells and 200 S. stipitis cells. Conversion factors between dry cell weight and number of cells were found by drying pure culture samples of each yeast after cell counts had been performed. These factors were found to be $0.006943 \mathrm{gdw} / \mathrm{L}$ for $S$. cerevisiae and $0.001944 \mathrm{gdw} / \mathrm{L}$ for S. stipitis. Ethanol, glucose and xylose concentrations were measured by YSI 2700 SELECT biochemistry analyzers (YSI Inc., Yellow Springs, OH) configured with the enzyme-bound membranes specified for each metabolite. Raw readings were interpreted by 2700 Xylose PC Software (YSI Inc., Yellow Springs, OH) to resolve cross-talk between the xylose and glucose specific membranes.

\section{Modeling}

The most comprehensive S. cerevisiae metabolic reconstruction currently available, iMM904 [55], was used for pure and mixed culture simulations. The fully compartmentalized network was reconstructed from 904 genes and accounts for 1228 metabolites and 1412 reactions. S. stipitis metabolism was simulated with iBB814 [20], the first published genome-scale reconstruction for this organism. This model accounts for 814 genes, 971 metabolites and 1371 reactions that are compartmentalized in the cytoplasm, mitochondria, and extracellular space. Following the publication of iBB814, a slightly more detailed S. stipitis metabolic reconstruction was developed [21]. We do not anticipate that the use of this alternative reconstruction would significantly alter the results reported in this paper.

The S. cerevisiae/S. stipitis co-culture model was constructed by combining the iMM904 and iBB814 stoichiometric matrices into a single matrix [56]. Flux distributions for S. cerevisiae $\left(v_{c}\right)$ and S. stipitis $\left(v_{s}\right)$ were calculated by solving the following linear program based on the assumption that the two species attempted to maximize their individual growth rates:

$$
\begin{gathered}
\max _{\mathrm{v}_{\mathrm{c}}, \mathrm{v}_{\mathrm{s}}} \mu=\mu_{\mathrm{c}}+\mu_{\mathrm{s}}=\mathrm{w}_{\mathrm{c}}^{\mathrm{T}} \mathrm{v}_{\mathrm{c}}+\mathrm{w}_{\mathrm{s}}^{\mathrm{T}} \mathrm{v}_{\mathrm{s}} \\
{\left[\begin{array}{cc}
\mathrm{A}_{\mathrm{c}} & 0 \\
0 & \mathrm{~A}_{\mathrm{s}}
\end{array}\right]\left[\begin{array}{l}
\mathrm{v}_{\mathrm{c}} \\
\mathrm{v}_{\mathrm{s}}
\end{array}\right]=\left[\begin{array}{l}
0 \\
0
\end{array}\right]} \\
{\left[\begin{array}{c}
\mathrm{v}_{\mathrm{c}, \min } \\
\mathrm{v}_{\mathrm{s}, \min }
\end{array}\right] \leq\left[\begin{array}{l}
\mathrm{v}_{\mathrm{c}} \\
\mathrm{v}_{\mathrm{s}}
\end{array}\right] \leq\left[\begin{array}{l}
\mathrm{v}_{\mathrm{c}, \max } \\
\mathrm{v}_{\mathrm{s}, \max }
\end{array}\right]}
\end{gathered}
$$

where the subscript $i$ represents the species, $A_{i}$ is the matrix of stoichiometric coefficients, $v_{i}$ is the vector of reaction fluxes including exchange fluxes, $v_{i, \min }$ and $v_{i, \max }$ are vectors of lower and upper flux bounds, $\mu_{i}$ is the growth rate, and $w_{c}$ and $w_{s}$ are vectors of experimentally determined weights that represent the contribution of each flux to biomass formation in S. cerevisiae [55] and S. stipitis [20], respectively. Other than competing for the common substrate glucose, the two yeasts were assumed to grow independently without species interactions. Therefore the co-culture objective function $\mu$ was assumed to be the sum of the individual species growth 
rates, and the inclusion of multi-level objective functions [34] was deemed unnecessary. The co-culture model was also used to simulate pure cultures of $S$. cerevisiae and $S$. stipitis by constraining all fluxes of the unmodeled organism to zero.

The steady-state flux balance model (2) was extended to a dynamic model through the addition of the following extracellular mass balance equations:

$$
\begin{aligned}
& \frac{d X_{c}}{d t}=\mu_{c} X_{c} \\
& \frac{d X_{s}}{d t}=\mu_{s} X_{s} \\
& \frac{d G}{d t}=-v_{g, c} X_{c}-v_{g, s} X_{s} \\
& \frac{d Z}{d t}=-v_{z, s} X_{s} \\
& \frac{d E}{d t}=v_{e, c} X_{c}+v_{e, s} X_{s} \\
& \frac{d O}{d t}=-v_{o, c} X_{c}-v_{o, s} X_{s}+k_{L} a\left(O^{*}-O\right)
\end{aligned}
$$

where $X_{c}$ and $X_{s}$ are the biomass concentrations of S. cerevisiae and S. stipitis, respectively, $G, Z$, and $E$ are the concentrations of glucose, xylose, and ethanol, respectively, $v_{e, \mathrm{c}}$ and $v_{e, \mathrm{~s}}$ are ethanol exchange fluxes, $v_{g, c}$ is the glucose uptake rate for $S$. cerevisiae, and $v_{g, s}$ and $v_{z, s}$ are the glucose and xylose uptake rates, respectively, for S. stipitis. An equation for the dissolved oxygen concentration $(O)$ was necessary to accurately describe microaerobic growth of S. stipitis (see results). In this equation (8), $v_{o, c}$ and $v_{o, s}$ are oxygen exchange fluxes, $k_{L} a$ is the volumetric mass transfer coefficient of oxygen from sparged gas to the culture medium, and $O^{*}$ is the saturation concentration of oxygen. For all simulations, $O^{*}$ was taken to be $0.24 \mathrm{mM}$, the saturation concentration for water at $30^{\circ} \mathrm{C}$ and $1 \mathrm{~atm}$.

The following substrate uptake expressions were used to calculate upper bounds on the actual sugar and oxygen uptake rates:

$$
\begin{aligned}
v_{g} & =v_{g, \max } \frac{G}{K_{g}+G} \frac{1}{1+\frac{E}{K_{\text {ieg }}}} \\
v_{z} & =v_{z, \max } \frac{Z}{K_{z}+Z} \frac{1}{1+\frac{G}{K_{i g z}}} \frac{1}{1+\frac{E}{K_{i e z}}} \\
v_{o} & =v_{o, \max } \frac{O}{K_{o}+O}
\end{aligned}
$$

where $v_{g, \max }, v_{z, \max }$ and $v_{o, \max }$ are the maximum uptake rates of each substrate, $K_{g}, K_{z}$ and $K_{o}$ are corresponding saturation constants, $K_{i e g}$ and $K_{i e z}$ are ethanol inhibition constants, and $K_{i g z}$ is a glucose inhibition constant. The glucose (9) and xylose (10) uptake rates were assumed to follow Michaelis-Menten kinetics with an additional inhibitory term that reflects growth rate suppression at high ethanol concentrations [57]. The glucose inhibition term added to the xylose uptake kinetics accounted for diauxic growth where S. stipitis favors glucose over xylose as the carbon source. The oxygen uptake rate was calculated from a Michaelis-Menten expression based on the dissolved oxygen content of the medium [15].

Pure and mixed culture dynamic flux balance models were solved using the Mosek optimization toolbox (Mosek ApS, Denmark) to resolve the linear program for intracellular metabolism within Matlab (Mathworks, Natick, MA) [30]. Because S. cerevisiae could not meet the non-growth associated ATP maintenance demand during the xylose-only consumption phase, the maintenance flux was constrained to zero after glucose depletion to prevent the LP solver from returning zero fluxes for the S. stipitis network. Due to time-scale differences between the sugar and oxygen consumption rates, the differential equation system (3)-(8) exhibited a high degree of stiffness. To reduce the time required to generate large numbers of DFBA simulations for parameter fitting and in silico culture optimization, Matlab stiff ODE solvers ode15s and ode23tb were used to obtain approximate solutions. An ODE solver with greater accuracy, ode23, was used to generate model predictions once parameters had been estimated or an optimum had been determined. A typical co-culture batch simulation that was solved in two minutes with ode15s required five hours with ode23.

\section{Abbreviations}

$\mu_{i}$ : Growth rate $(1 / \mathrm{h})$; $A_{i}$ : Stoichiometric Matrix; E: Ethanol concentration (g/L); G: Glucose concentration $(\mathrm{g} / \mathrm{L}) ; K_{g}$ : Glucose uptake saturation constant $(\mathrm{g} / \mathrm{L})$; $K_{i e}$ : Ethanol inhibition constant ( $\left.\mathrm{g} / \mathrm{L}\right) ; K_{\text {igz: }}$ Glucose inhibition constant $(\mathrm{g} / \mathrm{L})$; $k_{L} a$ : Oxygen mass transfer coefficient $\left(h^{-1}\right) ; K_{0}$ : Oxygen uptake saturation constant $(\mathrm{mM}) ; K_{z}$ : Xylose uptake saturation constant $(\mathrm{g} / \mathrm{L})$; $O$ : Dissolved oxygen concentration (mM); $O^{*}$ : Dissolved oxygen saturation concentration $(\mathrm{mM}) ; v_{c}:$ S. cerevisiae Flux distribution ( $\left.\mathrm{mmol} / \mathrm{gdw} / \mathrm{h}\right) ; v_{e, c}:$ S. cerevisiae ethanol flux (mmol/gdw/h); $v_{e, s}$ : S. stipitis ethanol flux (mmol/gdw/h); $v_{g, c}: S$. cerevisiae glucose uptake rate ( $\mathrm{mmol} / \mathrm{gdw} / \mathrm{h}) ; v_{\text {g, max }}$ : Maximum glucose uptake rate $(\mathrm{mmol} / \mathrm{gdw} / \mathrm{h}) ; v_{g, s}:$ S. stipitis glucose uptake flux (mmol/gdw/h); $v_{i, \text { max }}$ : Vector of upper bound flux constraints $(\mathrm{mmol} / \mathrm{gdw} / \mathrm{h}) ; v_{i, \min }$ : Vector of lower bound flux constraints ( $\mathrm{mmol} / \mathrm{gdw} / \mathrm{h}) ; v_{0, c}:$ S. cerevisiae oxygen uptake rate $(\mathrm{mmol} / \mathrm{gdw} / \mathrm{h}) ; v_{o, \text { max }}$ : Maximum oxygen uptake rate $(\mathrm{mmol} / \mathrm{gdw} / \mathrm{h}) ; v_{0, s}$ : S. stipitis oxygen uptake rate (mmol/gdw/h); $v_{s}$ : S. stipitis Flux distribution (mmol/gdw/h); $v_{z \text { max }}$ : Maximum xylose uptake rate $(\mathrm{mmol} / \mathrm{gdw} / \mathrm{h}) ; v_{z, s}:$ S. stipitis xylose uptake flux (mmol/gdw/h); $w_{c}:$ S. cerevisiae biomass reaction; $w_{s}$ : S. stipitis biomass reaction; $X_{c}: S$. cerevisiae concentration $(\mathrm{g} / \mathrm{L}) ; X_{s}: S$. stipitis concentration $(\mathrm{g} / \mathrm{L}) ; \mathrm{Z}$ : Xylose concentration $(\mathrm{g} / \mathrm{L})$.

\section{Competing interests}

The authors declare that they have no competing interests.

\section{Authors' contributions}

TH carried out the fermentation and modeling work and drafted the manuscript. MH conceived of the study, and participated in its design and coordination and helped to draft the manuscript. Both authors read and approved the final manuscript. 


\section{Acknowledgements}

This work was partially supported by the NSF-sponsored UMass Institute for Cellular Engineering IGERT program (Grant number DGE-0654128) and ReCommunity Recycling.

Received: 26 October 2012 Accepted: 12 March 2013

Published: 1 April 2013

\section{References}

1. Lynd LR, Weimer PJ, van ZyI WH, Pretorius IS: Microbial Cellulose Utilization: Fundamentals and Biotechnology. Microbiol Mol Biol Rev 2002, 66:506-577

2. Wyman CE: What is (and is not) vital to advancing cellulosic ethanol. Trends Biotechnol 2007, 25:153-157.

3. Lynd LR, Laser MS, Bransby D, Dale BE, Davison B, Hamilton R, Himmel M, Keller M, McMillan JD, Sheehan J, Wyman CE: How biotech can transform biofuels. Nat Biotech 2008, 26:169-172.

4. Gowen CM, Fong SS: Exploring Biodiversity for Cellulosic Biofuel Production. Chem Biodivers 2010, 7:1086-1097.

5. Gírio FM, Fonseca C, Carvalheiro F, Duarte LC, Marques S, Bogel-Łukasik R: Hemicelluloses for fuel ethanol: A review. Bioresour Technol 2010, 101:4775-4800.

6. Ha S-J, Galazka JM, Rin Kim S, Choi J-H, Yang X, Seo J-H, Louise Glass N, Cate JHD, Jin Y-S: Engineered Saccharomyces cerevisiae capable of simultaneous cellobiose and xylose fermentation. Proc Natl Acad Sci 2011, 108:504-509.

7. Pickens LB, Tang Y, Chooi Y-H: Metabolic Engineering for the Production of Natural Products. Ann Rev Chem Biomol Eng 2011, 2:211-236.

8. Almeida JRM, Runquist D, Sànchez Nogué V, Lidén G, Gorwa-Grauslund MF: Stress-related challenges in pentose fermentation to ethanol by the yeast Saccharomyces cerevisiae. Biotechnol J 2011, 6:286-299.

9. Brenner K, You L, Arnold FH: Engineering microbial consortia: a new frontier in synthetic biology. Trends Biotechnol 2008, 26:483-489.

10. Wei H, Xu Q, Taylor li LE, Baker JO, Tucker MP, Ding S-Y: Natural paradigms of plant cell wall degradation. Curr Opin Biotechnol 2009, 20:330-338.

11. Alper H, Stephanopoulos G: Engineering for biofuels: exploiting innate microbial capacity or importing biosynthetic potential? Nat Rev Micro 2009, 7:715-723

12. Eiteman MA, Lee SA, Altman R, Altman E: A substrate-selective cofermentation strategy with Escherichia coli produces lactate by simultaneously consuming xylose and glucose. Biotechnol Bioeng 2009, 102:822-827.

13. Fazzini RAB, Preto MJ, Quintas ACP, Bielecka A, Timmis KN, dos Santos VAPM: Consortia modulation of the stress response: proteomic analysis of single strain versus mixed culture. Environ Microbiol 2010, 12:2436-2449

14. Chemier JA, Fowler ZL, Koffas MAG: Trends In Microbial Synthesis of Natural Products and Biofuels. In Advances in Enzymology and Related Areas of Molecular Biology. John Wiley \& Sons, Inc; 2009:151-217.

15. Sonnleitner B, Käppeli O: Growth of Saccharomyces cerevisiae is controlled by its limited respiratory capacity: Formulation and verification of a hypothesis. Biotechnol Bioeng 1986, 28:927-937.

16. Matsushika A, Inoue H, Kodaki T, Sawayama S: Ethanol production from xylose in engineered Saccharomyces cerevisiae strains: current state and perspectives. Appl Microbiol Biotechnol 2009, 84:37-53.

17. Passoth $\mathrm{V}$, Zimmermann $\mathrm{M}$, Klinner U: Peculiarities of the regulation of fermentation and respiration in the crabtree-negative, xylose-fermenting yeast Pichia stipitis. App/ Biochem Biotechnol 1996, 57-58:201-212.

18. Silva JPA, Mussatto SI, Roberto IC, Teixeira JA: Fermentation medium and oxygen transfer conditions that maximize the xylose conversion to ethanol by Pichia stipitis. Renew Energy 2012, 37:259-265.

19. Shi NQ, Jeffries TW: Anaerobic growth and improved fermentation of Pichia stipitis bearing a URA1 gene from Saccharomyces cerevisiae. Appl Microbiol Biotechnol 1998, 50:339-345.

20. Balagurunathan B, Jonnalagadda S, Tan L, Srinivasan R: Reconstruction and analysis of a genome-scale metabolic model for Scheffersomyces stipitis. Microb Cell Fact 2012, 11:27.

21. Caspeta L, Shoaie S, Agren R, Nookaew I, Nielsen J: Genome-scale metabolic reconstructions of Pichia stipitis and Pichia pastoris and in silico evaluation of their potentials. BMC Syst Bio/ 2012, 6:24.
22. Delgenes JP, Escare MC, Laplace JM, Moletta R, Navarro JM: Biological production of industrial chemicals, i.e. xylitol and ethanol, from lignocelluloses by controlled mixed culture systems. Ind Crops Products 1998, 7:101-111.

23. Taniguchi M, Tohma T, Itaya T, Fujii M: Ethanol production from a mixture of glucose and xylose by co-culture of Pichia stipitis and a respiratorydeficient mutant of Saccharomyces cerevisiae. J Fermen Bioeng 1997, 83:364-370.

24. Srilekha Yadav K, Naseeruddin S, Sai Prashanthi G, Sateesh L, Venkateswar Rao L: Bioethanol fermentation of concentrated rice straw hydrolysate using co-culture of Saccharomyces cerevisiae and Pichia stipitis. Bioresour Technol 2011, 102:6473-6478.

25. Goldring ES, Grossman LI, Marmur J: Petite Mutation in Yeast. J Bacteriol 1971, 107:377-381.

26. Feist AM, Herrgard MJ, Thiele I, Reed JL, Palsson BO: Reconstruction of biochemical networks in microorganisms. Nat Rev Micro 2009, 7:129-143.

27. Price ND, Papin JA, Schilling CH, Palsson BO: Genome-scale microbial in silico models: the constraints-based approach. Trends Biotechnol 2003, 21:162-169.

28. Varma A, Palsson BO: Stoichiometric flux balance models quantitatively predict growth and metabolic by-product secretion in wild-type Escherichia coli W3110. Appl Environ Microbiol 1994, 60:3724-3731.

29. Mahadevan R, Edwards JS, Doyle lii FJ: Dynamic Flux Balance Analysis of Diauxic Growth in Escherichia coli. Biophys J 2002, 83:1331-1340.

30. Hiersted JL, Henson MA: Optimization of fed-batch Saccharomyces cerevisiae fermentation using dynamic flux balance models. Biotechno Progr 2006, 22:1239-1248.

31. Stolyar S, Van Dien S, Hillesland KL, Pinel N, Lie TJ, Leigh JA, Stahl DA: Metabolic modeling of a mutualistic microbial community. Mol Syst Biol 2007, 3:92

32. Zhuang K, Izallalen M, Mouser P, Richter H, Risso C, Mahadevan R, Lovley DR: Genome-scale dynamic modeling of the competition between Rhodoferax and Geobacter in anoxic subsurface environments. ISME J 2011, 5:305-316.

33. Salimi F, Zhuang K, Mahadevan R: Genome-scale metabolic modeling of a clostridial co-culture for consolidated bioprocessing. Biotech J 2010, 5:726-738.

34. Zomorrodi AR, Maranas CD: OptCom: A Multi-Level Optimization Framework for the Metabolic Modeling and Analysis of Microbial Communities. PLoS Comput Biol 2012, 8:e1002363.

35. Feist A: Bernhard: The growing scope of applications of genome-scale metabolic reconstructions using Escherichia coli. Nat Biotechnol 2008, 26:659-667.

36. Weierstall T, Hollenberg CP, Boles E: Cloning and characterization of three genes (SUT1-3) encoding glucose transporters of the yeast Pichia stipitis. Mol Microbiol 1999, 31:871-883.

37. Guijarro JM, Lagunas R: Saccharomyces cerevisiae does not accumulate ethanol against a concentration gradient. J Bacteriol 1984, 160:874-878.

38. Skoog K, Hahn-Hägerdal B: Effect of Oxygenation on Xylose Fermentation by Pichia stipitis. Appl Environ Microbiol 1990, 56:3389-3394.

39. Küenzi MT, Tingle MA, Halvorson HO: Sporulation of Saccharomyces cerevisiae in the Absence of a Functional Mitochondrial Genome. $J$ Bacteriol 1974, 117:80-88

40. Tzagoloff A, Dieckmann CL: PET genes of Saccharomyces cerevisiae. Microbiol Rev 1990, 54:211-225.

41. Hanly $T$, Urello $M$, Henson M: Dynamic flux balance modeling of $S$. cerevisiae and E. coli co-cultures for efficient consumption of glucose/ xylose mixtures. Appl Microbiol Biotechnol 2012, 93:2529-2541.

42. Neves AA, Vieira LM, Menezes JC: Effects of preculture variability on clavulanic acid fermentation. Biotechnol Bioeng 2001, 72:628-633.

43. Ligthelm ME, Prior BA, Preez JC, Brandt V: An investigation of $d$-xylose metabolism in Pichia stipitis under aerobic and anaerobic conditions. Appl Microbiol Biotechnol 1988, 28:293-296.

44. Soberón X: Saier Jr MH: Engineering Transport Protein Function: Theoretical and Technical Considerations Using the Sugar-Transporting Phosphotransferase System of Escherichia coli as a Model System. J Mol Microbiol Biotechnol 2006, 11:302-307

45. Kasahara T, Maeda M, Ishiguro M, Kasahara M: Identification by Comprehensive Chimeric Analysis of a Key Residue Responsible for High Affinity Glucose Transport by Yeast HXT2. J Biol Chem 2007, 282:13146-13150. 
46. Bertilsson M, Andersson J, Lidén G: Modeling simultaneous glucose and xylose uptake in Saccharomyces cerevisiae from kinetics and gene expression of sugar transporters. Bioprocess Biosyst Eng 2008, 31:369-377.

47. Taniguchi M, Tanaka T;: Clarification of Interactions among Microorganisms and Development of Co-culture System for Production of Useful Substances. 2004.

48. Postma E, Kuiper A, Tomasouw WF, Scheffers WA, van Dijken JP: Competition for glucose between the yeasts Saccharomyces cerevisiae and Candida utilis. Appl Environ Microbiol 1989, 55:3214-3220.

49. Laplace JM, Delgenes JP, Moletta R, Navarro JM: Effects of culture conditions on the co-fermentation of a glucose and xylose mixture to ethanol by a mutant of Saccharomyces diastaticus associated with Pichia stipitis. Appl Microbiol Biotechnol 1993, 39:760-763.

50. Albers E, Larsson C, Andlid T, Walsh MC, Gustafsson L: Effect of Nutrient Starvation on the Cellular Composition and Metabolic Capacity of Saccharomyces cerevisiae. Appl Environ Microbiol 2007, 73:4839-4848.

51. Heerde $E$, Radler F: Metabolism of the anaerobic formation of succinic acid by Saccharomyces cerevisiae. Arch Microbiol 1978, 117:269-276

52. Rieger M, Kappeli O, Fiechter A: The role of limited respiration in the incomplete oxidation of glucose by Saccharomyces cerevisiae. J Gen Microbiol 1983, 129:653-661.

53. Laplace JM, Delgenes JP, Moletta R, Navarro JM: Alcoholic fermentation of glucose and xylose by Pichia stipitis, Candida shehatae, Saccharomyces cerevisiae; and Zymomonas mobilis: oxygen requirement as a key factor. Appl Microbiol Biotechnol 1991, 36:158-162.

54. Lamping SR, Zhang H, Allen B: Ayazi Shamlou P: Design of a prototype miniature bioreactor for high throughput automated bioprocessing. Chem Eng Sci 2003, 58:747-758.

55. Mo M, Palsson B, Herrgard M: Connecting extracellular metabolomic measurements to intracellular flux states in yeast. BMC Syst Biol 2009, 3:37.

56. Hanly TJ, Henson MA: Dynamic flux balance modeling of microbial cocultures for efficient batch fermentation of glucose and xylose mixtures. Biotechnol Bioeng 2011, 108:376-385.

57. Hjersted JL, Henson MA, Mahadevan R: Genome-scale analysis of Saccharomyces cerevisiae metabolism and ethanol production in fedbatch culture. Biotechnol Bioeng 2007, 97:1190-1204.

doi:10.1186/1754-6834-6-44

Cite this article as: Hanly and Henson: Dynamic metabolic modeling of a microaerobic yeast co-culture: predicting and optimizing ethanol production from glucose/xylose mixtures. Biotechnology for Biofuels 2013 6:44

\section{Submit your next manuscript to BioMed Central and take full advantage of:}

- Convenient online submission

- Thorough peer review

- No space constraints or color figure charges

- Immediate publication on acceptance

- Inclusion in PubMed, CAS, Scopus and Google Scholar

- Research which is freely available for redistribution 